\title{
A Neural-Network Method for the Analysis of Multilayered Shielded Microwave Circuits
}

\author{
Juan Pascual García, Student Member, IEEE, Fernando Quesada Pereira, Student Member, IEEE, \\ David Cañete Rebenaque, Student Member, IEEE, José Luis Gómez Tornero, and \\ Alejandro Alvarez Melcón, Member, IEEE
}

\begin{abstract}
In this paper, a neural-network-based method for the analysis of practical multilayered shielded microwave circuits is presented. Using this idea, a radial basis function neural network (RBFNN) is trained to approximate the space-domain multilayered media boxed Green's functions used in the integral-equation (IE) method. Once the RBFNN has been trained, the outputs of the neural network (NN) replace the exact Green's functions, during the numerical solution of the IE. The computation of the RBFNN output values is very fast in comparison with the numerical methods used to calculate the exact Green's functions. This paper describes two novel strategies for efficiently training the RBFNN. In the first strategy, the input space of the RBFNN is divided into several spatial and frequency regions. The spatial subdivision is extended for the first time to both observation and source regions. In addition, the subdivision of the observation points regions is applied in a novel manner to the whole cross section of the metallic box. The second strategy combines the above region subdivision with an adaptive selection of the neurons variances in each region. The accuracy and the computational gain achieved with the NN method proposed makes possible the implementation of computer-aided-design tools that can be used for the analysis and design of integrated shielded microwave circuits (e.g., monolithic microwave integrated circuit devices) on a real-time basis.
\end{abstract}

Index Terms-Computer-aided design (CAD), multilayered circuits, multilayered Green's functions, neural networks (NNs), printed circuits, radial basis function neural network (RBFNN), shielded microwave circuits.

\section{INTRODUCTION}

$\mathbf{T}$ HE HIGH integration degree reached by modern microwave systems, especially with the advent of monolithic microwave integrated circuits (MMICs), makes the accurate analysis of these complex structures necessary [1]. This kind of microwave circuit is usually manufactured inside shielded enclosures, due to the isolation and mechanical support needed by these components. Nowadays, the industry demands useful tools for the design of microwave circuits with complex elements, which can operate at high frequencies, and with metallic walls surrounding the structures. Not only is accuracy in the calculation of the electrical behavior needed, but also fast computational times are desired in order to build useful computer-aided-design (CAD) packages [2]. The approximate

Manuscript received April 4, 2005; revised July 30, 2005. This work was supported in part under the Spanish National Project TEC2004-04313-C0202/TCM and under the Regional Seneca Project PB/4/FS/02.

The authors are with the Communications and Information Technologies Department, Technical University of Cartagena, Cartagena, Murcia E-30202, Spain (e-mail: juan.pascual@upct.es).

Digital Object Identifier 10.1109/TMTT.2005.861660 analysis techniques or quasi-static approaches can provide estimated results for simple structures in a fast way, but generally they do not achieve the analysis precision demanded nowadays for complex circuits. Therefore, full-wave and exact numerical methods have to be used for the analysis of complex microwave circuits.

Two of the techniques successfully applied for this purpose are the finite-element (FE) method and the finite-difference time-domain (FDTD) method. These two procedures allow for the exact analysis of practically any structure [3]. The main disadvantage of this family of methods is the large quantity of computer resources consumed when the complexity of the circuit increases. This is often due to the refinement of the mesh needed in complex structures to reach convergence in the solution.

Another popular method for the analysis of printed microwave circuits is the integral-equation (IE) approach, solved with the method of moments (MoM) [4]. In the IE technique, it is necessary to calculate the Green's functions associated with the multilayered shielded medium. In a shielded boxed media, the Green's functions are usually formulated as slow convergent spectral or spatial infinite series. Therefore, two possible formulations can be used in the IE solution. The spectral domain formulation is usually efficient, but, in some situations, the modal series shows a very slow convergence behavior, i.e., when the shielded box has large dimensions as compared to the printed circuit dimensions. Also, the convergence depends on the type of basis functions used, and the calculation of the required overlapping integrals can only be performed analytically with simple choices of basis functions [5].

The spatial domain formulation avoids the aforementioned problems of the spectral domain formulation. In this case, series acceleration techniques are employed to sum up the Green's functions in the spatial domain [6]. Once the Green's functions are obtained, the IE can be solved using any of the well-known spatial-domain discretization techniques [7]. However, the calculation of the associated series is still slow in order to obtain the desired electrical behavior in real time. Therefore, even though the IE combined with the MoM involves fewer unknowns than the FE or the FDTD, the method is still too slow to develop a CAD tool that operates in real time. This is now because of the time spent in the numerical calculation of the spatial-domain Green's functions. In this paper, we propose to use neural networks (NNs) to reduce the time needed for the computation of the spatial-domain Green's functions during the MoM solution of the IE technique. 
NNs can learn from a given data set. After this initial learning process called training, NNs produce fast outputs in classification and regression problems. This ability of learning the relation and structure of a multidimensional data in a nonlinear way has been exploited in many electromagnetic problems [8]. The first models of microwave components and circuits using NNs are found in [9]-[11]. NNs were also applied to microwave circuit optimization in [12] and [13]. After NNs proved their ability in microwave modeling and optimization, they were also used in the development of neural-based microwave CAD packages and library model tools [14], [15].

With respect to the analysis and modeling of printed circuits in layered media, we can find some different uses of NNs. NNs have been applied to the estimation of the coefficients needed in the discrete complex image method (DCIM) [16]. The DCIM originally appeared as an alternative technique to compute the spatial-domain Green's functions for a multilayered medium of infinite transverse dimensions [17]. In [16], the DCIM coefficients were calculated for a horizontal electric dipole placed at the top of a dielectric substrate over a specific bandwidth. Related to this, another technique was presented consisting on the employment of the NNs to evaluate the spectral domain integrals [18]-[20]. Consequently, the corresponding authors called this technique the neurospectral method. The input impedance of a microstrip antenna, with infinite dimensions in the transverse plane, was computed using this technique in [19]. Furthermore, in [20], the neurospectral method was employed for the design of a rectangular patch antenna, also with an infinite dielectric substrate.

More recently, NNs have been used in the estimation of the spatial-domain Green's functions of a shielded multilayered structure [21], [22]. In these works, the Green's functions were directly approximated by an NN for the case of a source and observation points placed inside the corresponding box, and for a specific fixed frequency. The NN method developed was later extended to the analysis of a practical microwave printed circuit in a given frequency range, in [23]. In that work, a strategy consisting on a subdivision of the spatial and frequency domains of the $\mathrm{NN}$ input space was carried out, in order to reduce the training and testing errors. The whole input space was divided into source point regions, observation point regions, and frequency regions. Each one of these regions was calculated according to the specific behavior of the Green's functions (e.g., singularity close to the source and resonant frequencies of the box). Originally, the subdivision in the source regions was only performed when a given observation point was placed in the nearest zone to the source point. For all other observation points, a single NN, without any source subdivision, was employed.

With respect to the training of the NNs, a technique employed in the past was to sample the input space with different points densities in regions where higher errors were detected [24]. In this paper we propose a new subdivision scheme which leads to improved precision in the Green's functions approximation. Now the subdivision in source regions is performed for all observation regions, except for the outermost observation region. There are two main advantages of the new proposed scheme. The first is that the enforcement of the boundary conditions for the potentials at the cavity walls is improved. The second is that the training set to each one of the new NNs is reduced.
In the above strategy, the selection procedure of the RBFNN variance remains fixed for all of the regions. A second novel strategy is developed in this paper to further reduce the generalization and training errors. In this new technique, an adaptive RBFNN variance algorithm is implemented. The selection of a suitable variance value, which can vary from observation region to observation region, allows a better approximation of the Green's functions, especially for the observation regions placed near the source point. Thanks to this novel strategy, more complex circuits with higher precisions can be analyzed. Both algorithms, once the RBFNNs have been trained, produce the Green's functions values needed in the IE technique in a very fast way. The time gain in the computational time makes the training time negligible, allowing for the analysis and optimization of shielded MMIC circuits in a near real-time fashion.

In Section II, the RBFNN model and the two new strategies are explained. In Section III, the analysis of three band-pass filters are discussed using the $\mathrm{NN}$ methods and the direct solution. The results show the improvement when the adaptive variance algorithm is used and the good agreement obtained between the exact solution and the neural method based solution. Measured results and simulations obtained with a spectral domain technique are also shown to validate the general CAD package.

\section{Proposed Method For NN Model}

The radial basis function network (RBFNN) is one kind of NN composed of three fixed layers. The input layer takes the data from the input space. The hidden layer transforms the input space nonlinearly in a hidden space of higher dimensionality. Finally, the output layer performs a linear mapping. In our problem, the RBFNN must approximate the Green's functions values. This approximation task is a regression problem. The RBFNN with a single hidden layer of Gaussian neurons are considered to be universal approximators [25]. Therefore, we choose the Gaussian function as the nonlinear function of each neuron. The total transformation made by the RBFNN is shown in the following equation:

$$
F(\mathbf{x})=b+\sum_{k=1}^{M} w_{k} \cdot \varphi\left(\left\|\mathbf{x}-\mathbf{c}_{k}\right\|\right)
$$

where $\varphi$ is a nonlinear function. The nonlinear function is the Gaussian function just mentioned, described as follows:

$$
\varphi\left(\left\|\mathbf{x}-\mathbf{c}_{k}\right\|\right)=\exp \left(-\frac{1}{2\left(\mathbf{x}-\mathbf{c}_{k}\right)^{T} \mathbf{C}^{T} \mathbf{C}\left(\mathbf{x}-\mathbf{c}_{k}\right)}\right)
$$

and the variance values are cast in the following matrix:

$$
\mathbf{C}=\left(\begin{array}{cccc}
\frac{1}{\sigma_{1}} & 0 & \ldots & 0 \\
0 & \frac{1}{\sigma_{i}} & \ldots & 0 \\
\vdots & \vdots & \ddots & \vdots \\
0 & 0 & 0 & \frac{1}{\sigma_{n}}
\end{array}\right)
$$

where $\mathbf{c}_{k}$ is the center of the $k$ th radial basis function, $\mathbf{x}$ is the input vector, $b$ is a constant or bias, $w_{k}$ is the weight of the $k$ th neuron, $\mathbf{C}$ is the norm weighting matrix, $n$ is the input space 


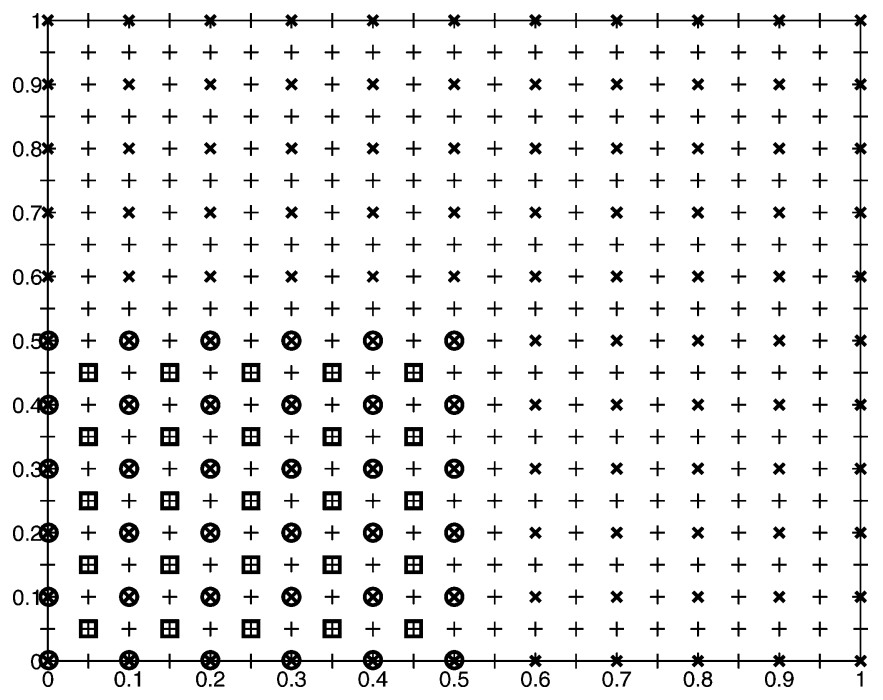

Fig. 1. Distribution of source and observation points of the training and the testing sets. The observation points of the training set are the crosses; the source points of the training set are the circles; the source points of the testing set are the square points, and the observation points of the testing set are the pluses points. In this distribution, there is not any subdivision in the input space.

dimension, and $M$ is the total number of neurons. The operator $\|\cdot\|$ denotes the Euclidean norm. Using the norm weighting matrix, it is possible to define one particular variance value $\sigma$ associated with each one of the input space coordinates, as indicated in (3).

The structure of the RBFNN was introduced for the first time in [23]. In our problem, each input space point is composed of the transverse spatial coordinates of a source point $\left(x^{\prime}, y^{\prime}\right)$, the transverse spatial coordinates of the observation point $(x, y)$, and the frequency. The corresponding output space is composed of the real and imaginary parts of the relevant mixed-potential Green's functions $\left(G_{v}, G_{a x}\right.$, and $\left.G_{a y}\right)$. The selection of a variance matrix, as shown in (3), instead of a unique variance for all of the coordinates, is especially important in our problem, due to the characteristics of the input space. The first four coordinates are spatial coordinates, while the fifth coordinate is the frequency of operation. In general, a different variance can be selected for each one of the input coordinates. Each of the spatial coordinates in normalized form range from zero to one, as can be seen in Fig. 1. The frequency of operation expressed in gigahertz can range typically from 3 to 30 to cover many practical microwave and millimeter-wave applications. All of the studies made have shown that a suitable variance for the spatial coordinates must be different from the variance for the frequency coordinate for the best performance.

The RBFNN design involves two steps: the training stage and the testing stage. During the training phase, the centers, weights, and variances are calculated. To compute these parameters, a training set of input and output data pairs is needed. A typical distribution of source and observation points of the training and testing sets is shown in Fig. 1. The source and observation points of the testing set are placed at intermediate positions with respect to the source and observation points of the training set. This testing point distribution allows to check the generalization and interpolation capabilities of the RBFNN. For the training

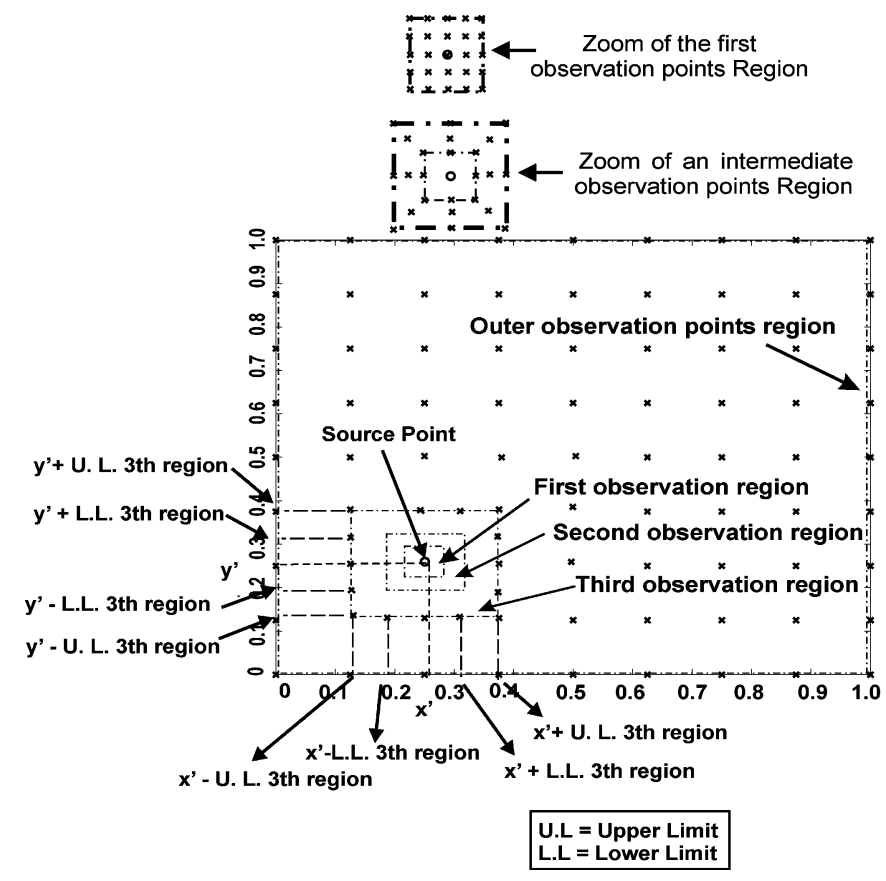

Fig. 2. Subdivision of the input space in different observation regions.

stage, the Green's functions are computed in the spatial domain following the numerical techniques described in [6] and [26].

In the distribution shown in Fig. 1, there is no region subdivision in the input space. Similar distributions were used in [22] and [21]. Due to problem symmetries, the source points are distributed along a spatial net belonging to the first quadrant of a rectangular box of dimensions $(a, b)\left(x^{\prime}<=a / 2\right.$, $y^{\prime}<=b / 2$ ); see Fig. 1 [21]. The Green's functions values associated with the remainder source points placed at the other quadrants are calculated from the corresponding source points lying in the first quadrant by straightforward symmetry. Among the different RBFNN training algorithms, we have chosen the orthogonal least squares (OLS) procedure [27], [28]. Although the OLS is not capable of providing the most compact set of centers, as shown in [29], it has been chosen because it surpasses other training algorithms in the Green's functions approximation problem, as described in [21].

\section{A. First Strategy: Automatic Region Subdivision With Fixed Variance}

The objective of the method proposed is to use the RBFNN as a substitute of the exact method to compute the Green's functions. Although the singularity is properly extracted [6], the Green's functions exhibit rapid variations near the source in a totally closed environment. The RBFNN, like other NNs, cannot approximate fast variations in an appropriate way. One useful strategy to overcome this problem is to apply the divide-andconquer principle. Thus, the observation points are grouped in different areas depending on their proximity to the source point (see Fig. 2).

The procedure to implement the subdivision of observation regions is shown in Fig. 3. Initially, the algorithm selects a first observation region. This first region ranges from the source point to an initial limit. The mentioned initial limit can be 


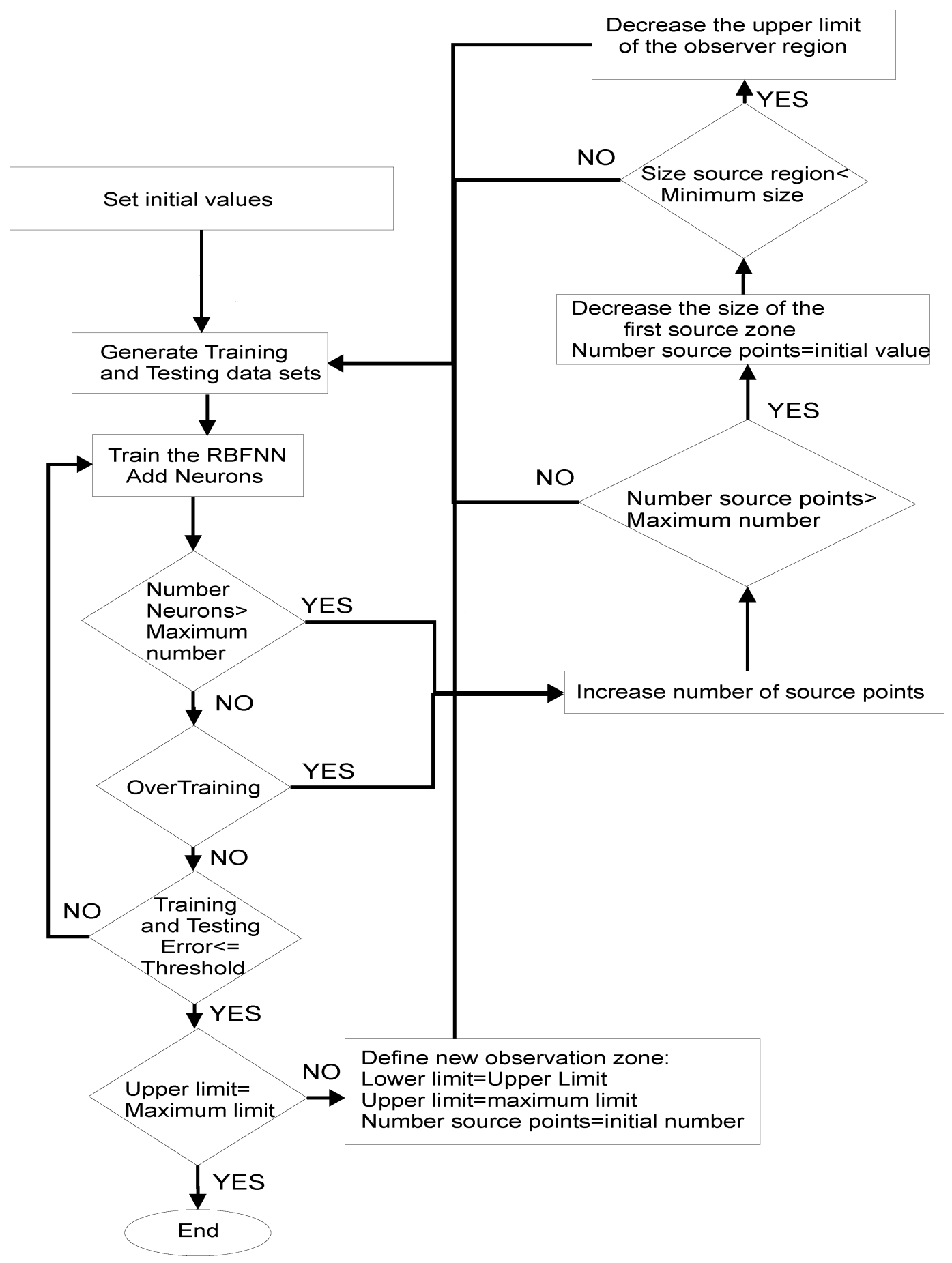

Fig. 3. Flowchart of the observation points region subdivision algorithm.

computed as a fraction of the distance between source points in a regular net filling the first quadrant of the box (see Fig. 1). In a similar way, the maximum limit can be chosen to be equal to the distance between source points in this net. Also, the frequency is set to the highest value of the given bandwidth. The Green's functions show faster variations at higher frequencies. Consequently, computing the observation regions at this maximum frequency will assure a correct training for all of the other frequency points. In addition, some other parameters must be initialized before the algorithm can start, such as the variance and the maximum limits for the observation and first source regions.
It is important to point out that the observation regions close to the source are smaller in size, while the number of observation points is similar in all regions. In this way, the density of observation points is larger for regions close to the source, therefore increasing the accuracy of the NN. In Fig. 2, we can see the distribution of the observation points in the first observation region (i.e., the one placed closest to the source point). The figure also shows the observation points distribution in an intermediate region. The first region is filled with a set of observation points that are regularly distributed. All other intermediate regions have the same distribution of observation points, as shown in Fig. 2. 
Finally, the training and testing sets are generated with the exact Green's functions routines, based on the algorithms described in [6] and [26]. Neurons are added until the error threshold for the training and testing set is reached or until an overtraining situation is detected. Overtraining situations appear when the testing error suddenly grows. If overtraining occurs or the number of neurons surpasses the maximum number allowed, the number of source points is increased. If the number of source points cannot be increased (for instance, due to memory limitations), then the size of the first source region is decreased. This feedback operation leads to a more dense net of source and observation points, so that the training is improved. If the mentioned operations are not successful, the upper limit of the observation region is decreased (see Fig. 3). After the selection of the first observation zone is completed, the selection of the next observation region begins. The upper limit becomes the maximum limit, and the lower limit becomes the upper limit of the previous zone. The algorithm continues computing the sizes of the regions until the final upper limit of a given region coincides with the maximum limit. In this case, the subdivision in observation regions stops, and control is given to the subdivision in the source regions. The source region subdivision is a new concept introduced in this paper.

The first advantage in performing a subdivision in source regions is that the boundary conditions for the potentials at the cavity walls are enforced more easily. The points placed near the walls are separated from the ones placed far from the walls, as shown in Fig. 4. This separation allows for the enforcement of the boundary conditions more efficiently. In addition to the better fulfillment of the boundary conditions, an interesting motivation to perform a subdivision in source regions is the need to limit the size of the training and testing sets. If no source region subdivision is made, a regular net of source points filling the first quadrant of the box is needed. The problem with this choice is that the training set would be too large to train the RBFNN properly, i.e., a net of $15 \times 15$ source points with 25 observation points per source would generate 5625 input points per frequency. The training stage would consume too much in terms of computer resources, running time, and memory allocation. This problem is avoided using the new concept of subdivision in source regions.

To start with the source region algorithm, we have to remark that the previous observation region selection procedure also computes the first source region, as can be seen in the flow chart shown in Fig. 3. The source region subdivision algorithm begins with the setting of some necessary initial parameters. The frequency is set to the highest value of interest due to the same reasons mentioned before. Also, the variance is set to the same fixed value of the previous algorithm. The RBFNN training begins after the generation of the training and testing sets. If an overtraining situation is detected or the maximum number of neurons is exceeded, the number of source points is increased. If the error reaches the preset threshold, a new source region is created, as shown in Fig. 5. In [23], the source region subdivision was computed only for the first observation region, namely for the observation points placed nearest to the corresponding source point. The training and testing sets for the rest of the observation points were computed using similar uniform nets, as

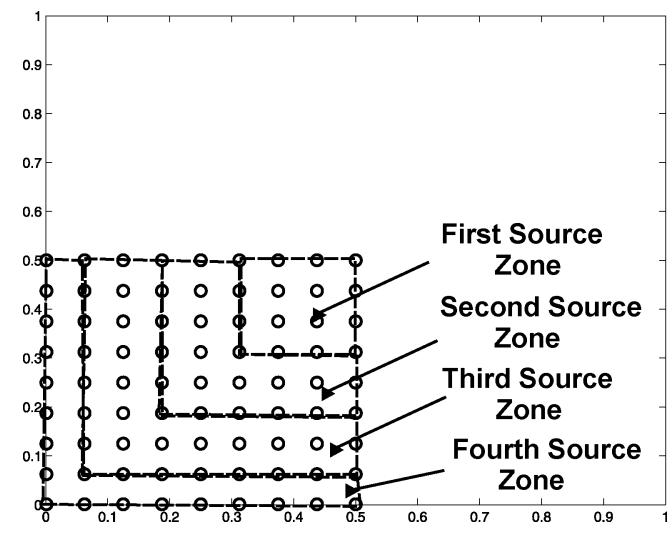

Fig. 4. Subdivision in different source regions.

shown in Fig. 1. In this paper, the idea is generalized, and now the source region division is extended for all of the observation regions. This subdivision is more complex than the one used in [23], but leads to an $\mathrm{NN}$ which improves the fulfillment of the boundary conditions at the cavity walls. Also, with the new algorithm, a better approximation of the Green's functions is achieved in the square box, near and far from the metallic walls.

It is important to remark that the division in source regions is performed in a way so that all regions have similar areas. In addition, the number of source points in all regions is similar, thus resulting in a similar density of source points in all regions. This strategy is different than the one followed for the observation regions. This is because now the behavior of the Green's function is not abruptly close to the walls. The important point to construct the source regions is to adopt the " $\mathrm{L}$ "-shaped areas shown in Fig. 4. These areas have shown to be appropriate to more efficiently separate the points close to the walls from the points placed far from the walls.

Following the same example of a net of $15 \times 15$ source points, we can generate five source regions, with each one containing 45 source points. If the number of observation points in a given observation region is of 25 , then the total size of the training set will be 1125 per each frequency point. On the contrary, if no source region division is performed, the total size of the training set would be 5625 points. Due to memory limitations, it would be difficult to handle a training set of 5625 points for each frequency.

A related problem is if we find an overlearning situation when dealing with very small training sets. If this happens, the technique offers enough flexibility to modify the number of points in a given source region. For instance, we can take 100 points in a source region, therefore leading to a training set of 2500 points. This can eliminate the overlearning situation problem, while still maintaining a reasonable training set size.

Finally, a third division is made at the frequency level of the input space. The division at the frequency level was introduced and discussed in [23]. The first motivation of introducing the frequency division is again to reduce the size of the training set. A second motivation, as recognized in [23], is to properly handle the resonant frequencies that can occur inside shielded boxes. In [23], a low-pass filter was analyzed having one resonant frequency inside the bandwidth of interest. The results presented showed the accuracy obtained and confirmed that resonances 


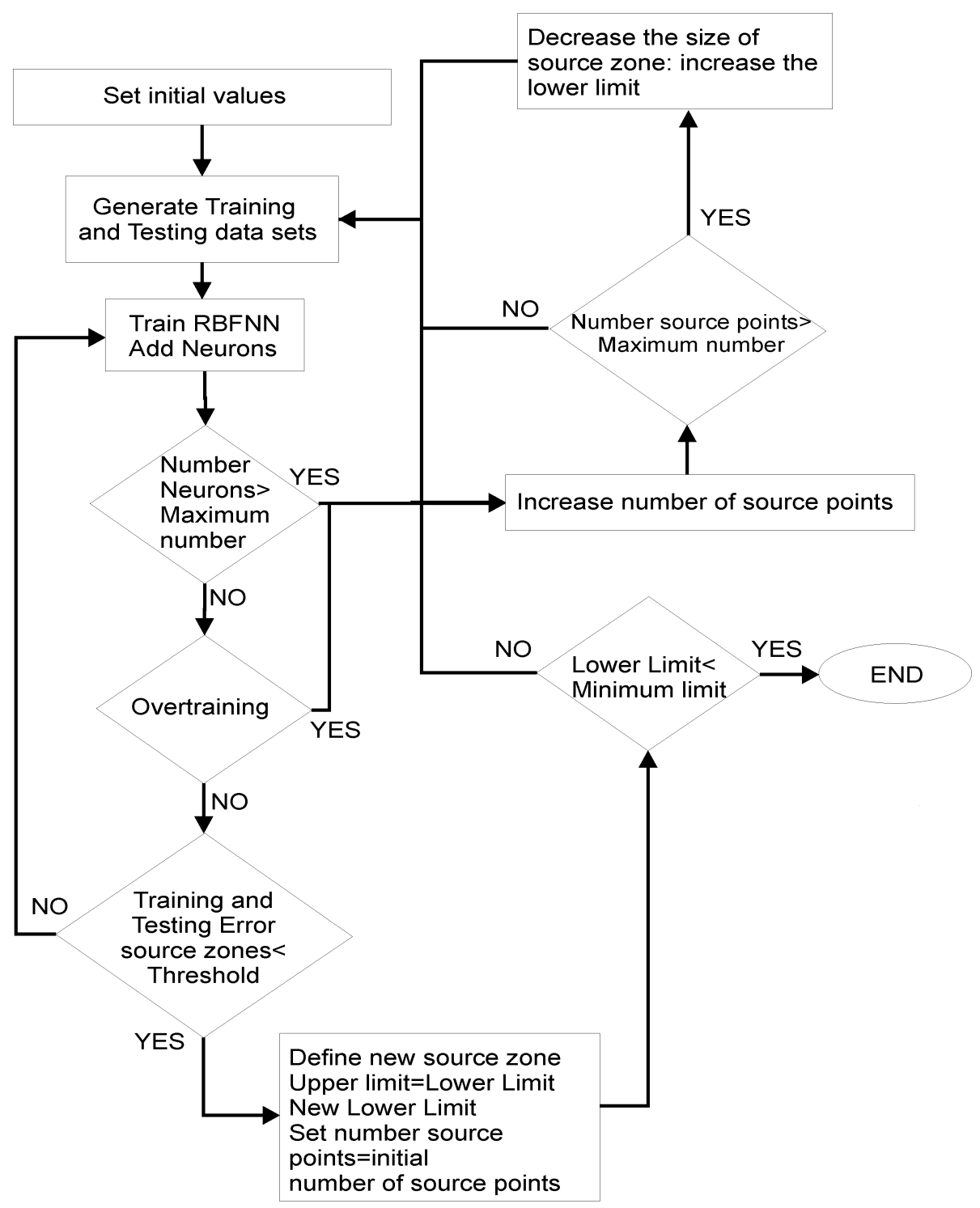

Fig. 5. Flowchart of the source points region subdivision algorithm.

can be properly treated with the frequency division strategy without loss of accuracy.

\section{B. Second Strategy: Region Subdivision \\ With Adaptive Variance}

In this second strategy, the region subdivision method is completed with a mechanism that finds, in an adaptive fashion, a suitable Gaussian variance. In [21] and [22], a study of some appropriate variance values, associated with the OLS training algorithm, was presented. Although a range of variance values exists that permits a correct training, a norm weighting matrix filled with inappropriate values will increase both the training and the testing errors. The optimum variance value is related to the distribution and to the distance between the input space points. Also, it is linked with the frequency content of the output data. Neurons with lower variance values are needed if the output data present high-frequency content. For a fixed distance between the input data points, there is a minimum value of variance that guarantees the generalization and inter- polation abilities of the RBFNN. Smaller values of the variance would lead to large generalization errors. In this case, the only solution in order to decrease the error is to increase the number of training points.

The Green's functions in a totally shielded box exhibit fast variations near the source, and, therefore, low values of variance are needed. On the other hand, far from the singularity, the variations are smoother, and thus higher values of variance would be more convenient. In the previous strategy, a fixed initial variance is selected within a reasonable range of variances as reported in [22]. However, this value is not the optimum for all source-observation distances. If the variance is fixed constant throughout the whole training process, a large number of observation and source regions would be needed to achieve a given target precision. Thus, an adaptive mechanism to find an appropriate variance value is developed in this paper. The adaptive method is applied inside the observation region algorithm. Once the observation regions and the first source region have been selected, the variance remains fixed for the rest of the source regions. Con- 
sequently, each observation region will have associated its own optimum variance value.

It should be noted that the spatial variance is referred to the first four values of the diagonal norm weighting matrix of (3). The first four values correspond to the spatial coordinates and the fifth value to the frequency coordinate. We have found that a clever choice is to set all spatial variances to the same value: $\sigma_{1}^{2}=\sigma_{2}^{2}=\sigma_{3}^{2}=\sigma_{4}^{2}=\sigma_{s}^{2}$. Consequently, a different variance value is selected for the frequency coordinate $\left(\sigma_{f}\right)$. We have to keep in mind that the adaptive variance method developed operates on the unique value for the spatial variance: $\sigma_{s}$.

The initial variance value $\left(\sigma_{s}\right)$ is set at the beginning of the observation region algorithm. This initial value must be selected within some variance limits [22]. If the training and testing threshold error is not reached, the variance is decreased at each step. If the error is still above the threshold, the variance is decreased again, until the RBFNN is successfully trained or the minimum variance is reached. If this last situation is detected, a new variance increment phase begins.

If an optimum variance value is not obtained following this search procedure, then either the number of source points is increased or the size of the first source region is decreased. In the algorithm, both situations are explored sequentially.

As the results in Section III will show, the adaptive variance method outperforms the fixed variance method. On the one hand, lower error levels are obtained, and, on the other hand, a smaller number and larger observation regions are needed to achieve the same error level.

\section{RESULTS}

In order to demonstrate the usefulness of the proposed neural strategies, we have carried out two numerical tests. In the first one, we have measured the size of the training set versus the normalized mean square error (NMSE). We compare the sizes obtained with the new strategy extended to all observation regions developed in this paper, with respect to the direct strategy implemented in [23].

First, one observation region was selected with upper limit equal to 0.0208 and lower limit equal to 0.0104 in the shielded box structure shown in Fig. 6. Without any source subdivision, the NMSE achieved for the given observation zone is 0.48 . The size of the training set is not allowed to be enlarged due to memory limitations (a maximum of 4059 in this example); thus, it is impossible to reach lower NMSE levels. On the contrary, when the source regions subdivision is applied, the mean of the size of the training set for each source region drops to 2662, and the NMSE error falls below the 0.20 level. A lower error is obtained with a smaller training set size.

As a second numerical test, we want to evaluate if the observation region subdivision is more efficiently carried out when the adaptive variance strategy is applied. The numerical test is performed on the same box structure shown in Fig. 6. We have found that, when the error level (NMSE) is large, essentially the same number of source points and number of observation regions are needed in both fix and adaptive algorithms. However, when the NMSE decreases below 0.2, the adaptive algorithm needs a smaller training set (e.g., from 576 to 484 , representing a $26 \%$ gain). The adaptive variance method then permits a smaller

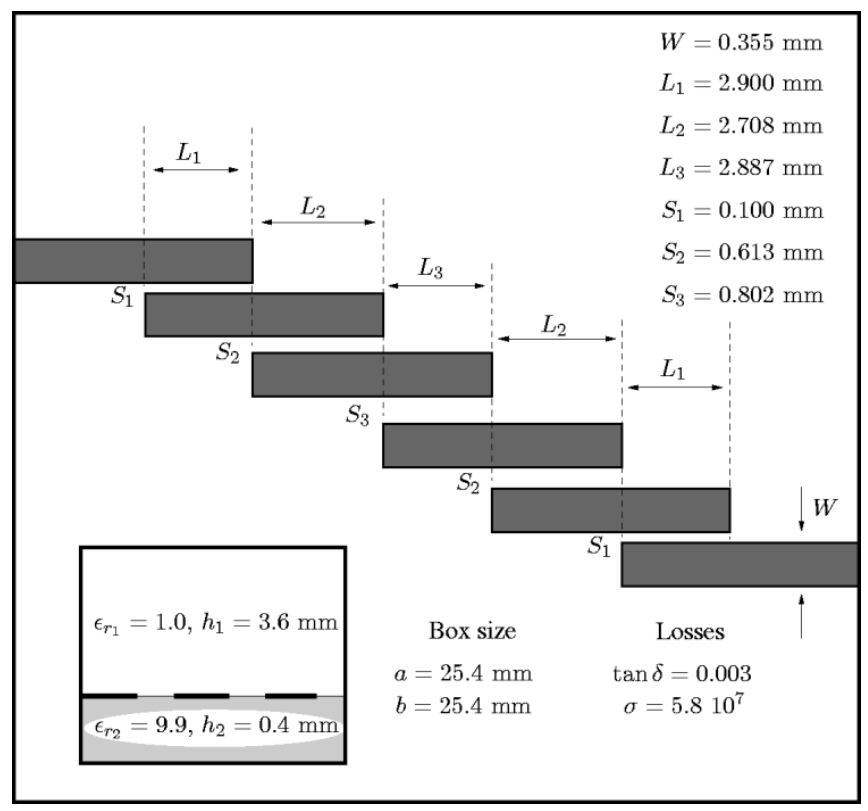

Fig. 6. Shielded bandpass printed filter 1 . The structure of the filter and the dimensions of the box are included.

training set, improving the overall efficiency of the training. In a similar way, it also permits to obtain lower errors for a given fixed training set size.

Two different bandpass filters have been analyzed using the $\mathrm{NN}$ strategies. A comparison of the scattering parameters calculated using the NN method and the direct Green's functions is shown in this section. As stated in the previous sections, all of the RBFNNs of the corresponding spatial and frequency regions are trained for a specific box and layered structure (see Fig. 6).

After this initial phase, the exact Green's functions are replaced by the RBFNN outputs, during the numerical solution of the IE. The corresponding RBFNN will compute in a fast way the output values, reducing the computational time drastically. Once the RBFNNs have been trained to substitute the Green's functions (for a fixed shielded box and for a given bandwidth), any printed circuit inside the same box can be analyzed or optimized quite efficiently. The training time is a constant initial quantity. After that, any circuit analysis or optimization task can be carried out without the need to retrain the structure.

The region subdivision with fixed variance strategy and the region subdivision with adaptive variance strategy, introduced in this paper, were applied to the multilayered shielded box shown in Fig. 6. The frequency bandwidth ranges from 9 to $11 \mathrm{GHz}$.

In the region subdivision with fixed variance, the value of the spatial variance was set to a fixed value of $\sigma_{s}^{2}=0.05$, according to the studies presented in [23]. With this value, this strategy reaches a minimum NMSE of 0.2. The observation subdivision algorithm produced three spatial observation regions, as shown in Table I. Moreover, the source subdivision algorithm generated six spatial regions (see Table II). The lower and upper limits in Tables I and III are referred to the limits of the corresponding observation zones, as shown in Fig. 2. The observation points that are not included in these observation zones belong to the outermost observation region.

For this example, the frequency region subdivision algorithm divides the given bandwidth into only one region that ranges 
TABLE I

OBSERVATION REGIONS GENERATED BY THE REGION SUBDIVISION WITH FIXED VARIANCE

\begin{tabular}{|c||c|c|}
\hline Region & Lower Limit & Upper Limit \\
\hline \hline 1st Region & Source point position & 0.0208 \\
\hline 2nd Region & 0.0208 & 0.0625 \\
\hline 3th Region & 0.06125 & 0.125 \\
\hline
\end{tabular}

TABLE II

SOURCE REGIONS GENERATED BY THE REGION SUBDIVISION WITH FIXED VARIANCE

\begin{tabular}{|c||c|c|}
\hline Region & Lower Limit & Upper Limit \\
\hline \hline 1st Region & 0.3 & 0.5 \\
\hline 2nd Region & 0.2 & 0.3 \\
\hline 3th Region & 0.125 & 0.2 \\
\hline 4th Region & 0.0688 & 0.125 \\
\hline 5th Region & 0.0266 & 0.0688 \\
\hline 6th Region & 0.001 & 0.0266 \\
\hline
\end{tabular}

from 9 to $11 \mathrm{GHz}$. Only three frequency points were needed to achieved the accuracy demanded $(9,10$, or $11 \mathrm{GHz})$, selecting a variance for the frequency coordinate of: $\sigma_{f}^{2}=10$. The use of this simple frequency division scheme is possible because of the absence of resonant frequencies of the box in the bandwidth of interest. The benefits of the frequency region subdivision were discussed in more detail in [23]. In that paper, a low-pass filter was analyzed in a frequency range containing one resonance of the box. In [23], it is shown how the frequency region subdivision can correctly handle resonant frequencies of the box without loss of accuracy.

After generating the observation, source, and frequency regions, the RBFNN training begins. The training procedure has to be applied to all combinations of source, observation, and frequency regions. In this particular case, each RBFNN approximates the Green's functions throughout the whole bandwidth, due to the fact that only one frequency region was generated.

We have also applied the region subdivision with adaptive variance strategy to the same layered structure. With an initial spatial variance of $\sigma_{s}^{2}=0.05$, the lowest NMSE error level reached was of only 0.10 . It can be seen that, with this adaptive scheme, the error level that can be obtained is lower than that with the previous strategy. It should be pointed out that, with the fixed variance strategy, the NMSE error could not be lowered from the 0.2 level. Therefore, better precision is expected when the adaptive variance algorithm is used. To reach this error level, the subdivision algorithm produced six observation regions (see Table III).

The spatial variance was automatically reduced by the algorithm from the initial value of 0.05 up to a value as low as 0.01 for the case of the first region. The change in the value of the variance for all other regions is detailed in Table III. This change in the spatial variance permits a more precise observation region selection, extending the generalization capabilities of the RBFNN in the different regions. Consequently, lower NMSE levels are obtained. After the observation region subdivision, the source region subdivision produced 12 regions (see Table IV).

The filter shown in Fig. 6 was analyzed using the fixed variance strategy, the adaptive variance strategy, and the direct solution of the Green's functions. In Figs. 7 and 8, the module
TABLE III

OBSERVATION REGIONS GENERATED BY THE REGION SUBDIVISION WITH ADAPTIVE VARIANCE

\begin{tabular}{|c||c|c|c|}
\hline Region & Lower Limit & Upper Limit & Variance $\sigma^{2}$ \\
\hline \hline 1st Region & Source point position & 0.0052 & 0.01 \\
\hline 2th Region & 0.0052 & 0.0104 & 0.025 \\
\hline 3nd Region & 0.0104 & 0.0208 & 0.05 \\
\hline 4nd Region & 0.0208 & 0.0417 & 0.05 \\
\hline 5th Region & 0.0417 & 0.0625 & 0.05 \\
\hline 6th Region & 0.0625 & 0.125 & 0.05 \\
\hline
\end{tabular}

TABLE IV

SOURCE REgIONS GENERATED By THE REGION SUBDIVISION WITH ADAPTIVE VARIANCE

\begin{tabular}{|c||c|c|}
\hline Region & Lower Limit & Upper Limit \\
\hline \hline 1st Region & 0.4 & 0.5 \\
\hline 2nd Region & 0.3 & 0.4 \\
\hline 3nd Region & 0.2 & 0.3 \\
\hline 4th Region & 0.1438 & 0.2 \\
\hline 5th Region & 0.1200 & 0.1438 \\
\hline 6th Region & 0.1022 & 0.1200 \\
\hline 7th Region & 0.0844 & 0.1022 \\
\hline 8th Region & 0.0669 & 0.0844 \\
\hline 9th Region & 0.0491 & 0.0669 \\
\hline 10th Region & 0.0313 & 0.0491 \\
\hline 11th Region & 0.0135 & 0.0313 \\
\hline 12th Region & 0.001 & 0.0135 \\
\hline
\end{tabular}

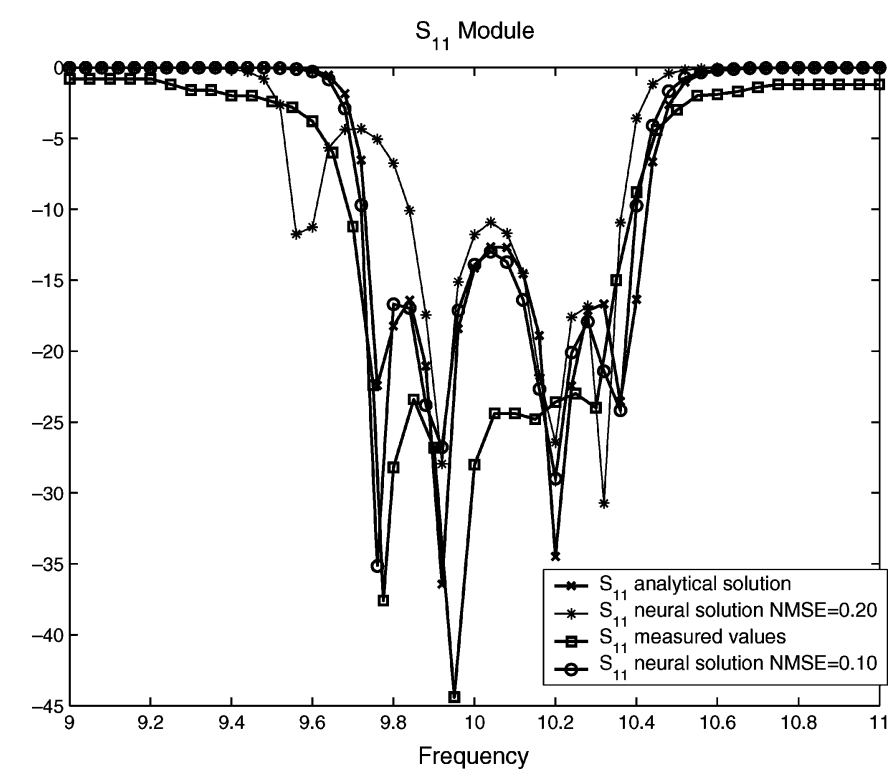

Fig. 7. $S_{11}$ module for the filter shown in Fig. 6. Results are shown for the direct solution, for the $\mathrm{NN}$ method with fixed variance solution (NMSE = 0.20 ), for the NN method with adaptive variance solution (NMSE $=0.10$ ), and for measurements.

of the $S_{11}$ and $S_{21}$ parameters calculated using the three techniques are presented. The results show the increased accuracy achieved by the adaptive variance strategy with respect to the fixed variance strategy. Measured results are also shown to confirm the validity of the proposed technique. 


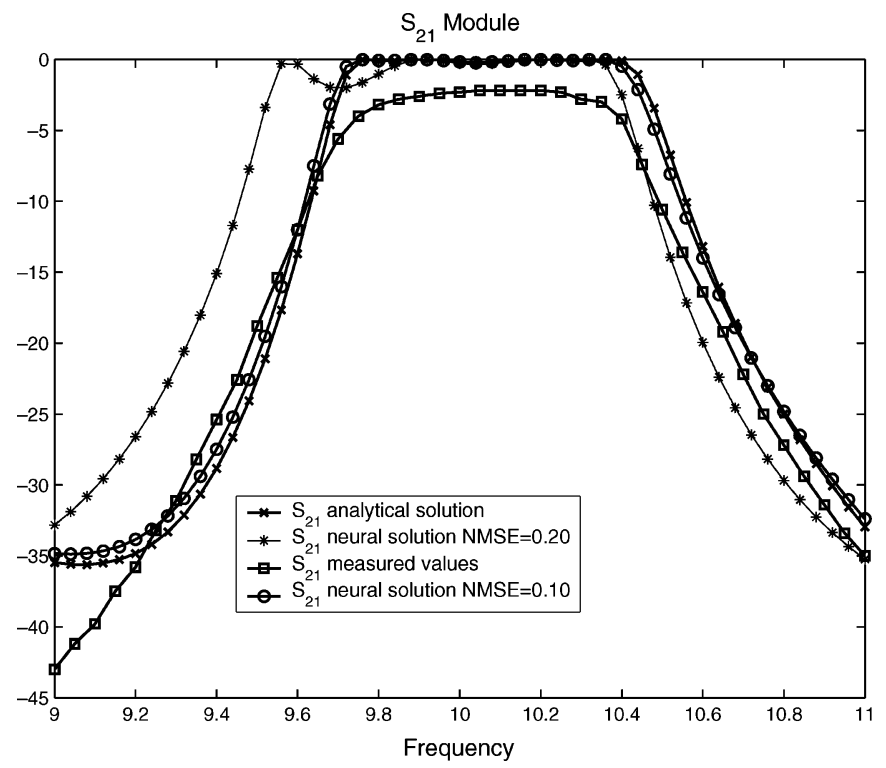

Fig. 8. $S_{21}$ module for the filter shown in Fig. 6. Results are shown for the direct solution, for the NN method with fixed variance solution (NMSE = 0.20 ), for the $\mathrm{NN}$ method with adaptive variance solution (NMSE $=0.10$ ), and for measurements.

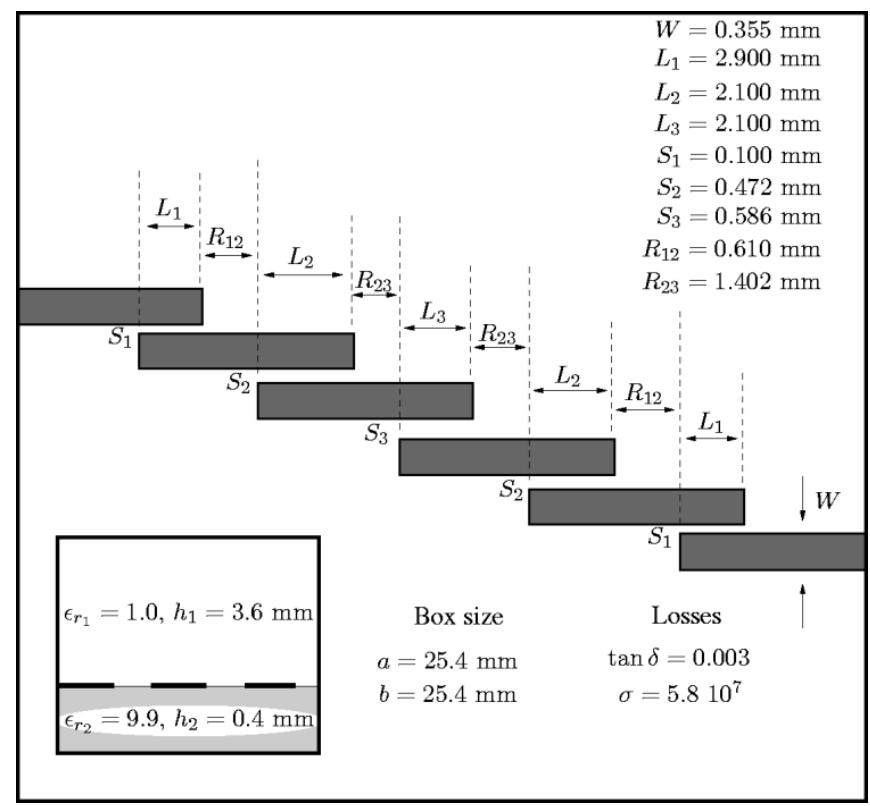

Fig. 9. Shielded bandpass printed filter 2. The structure of the filter and the dimensions of the box are included.

It should be pointed out that the precision attained with the technique originally proposed in [23] is not enough to obtain meaningful results for the filter shown in Fig. 6. This is due to the presence of very critical coupling gaps between the printed lines of the structure. The two main extensions presented in this paper allow for the accurate analysis of this bandpass filter. First, the extension of the source region division for more than one observation region leads to further reduction of the overall RBFNN error. Second, the adaptive variance algorithm allows for the design of an RBFNN with improved generalization capabilities.

A second filter placed inside the same metallic box was analyzed using the direct solution and using the two mentioned

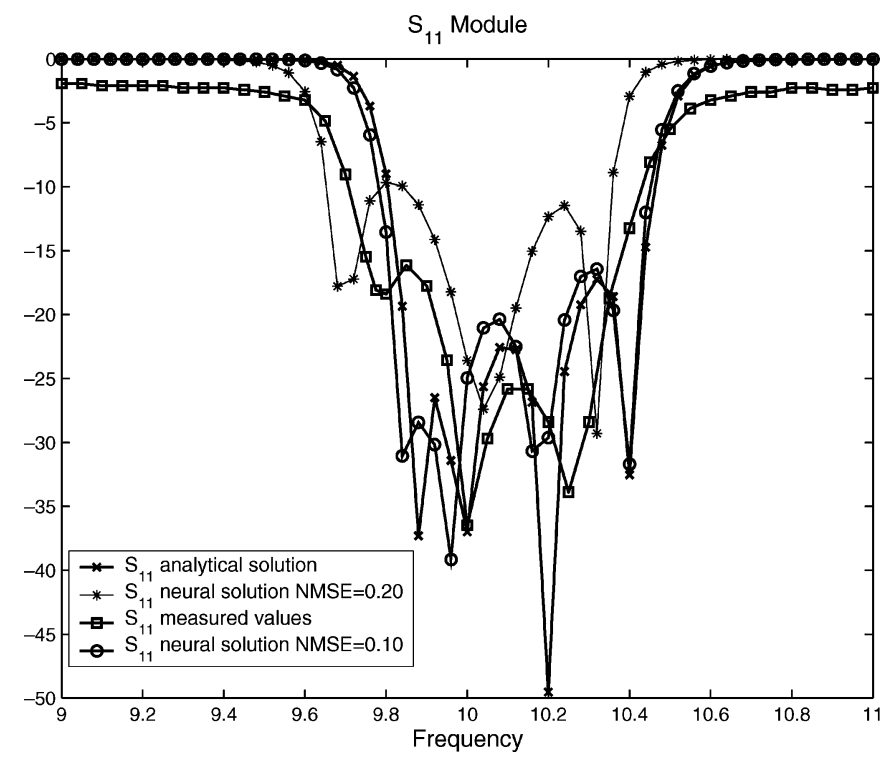

Fig. 10. $S_{11}$ module for the filter shown in Fig. 9. Results are shown for the direct solution, for the NN method with fixed variance solution (NMSE = 0.20 ), for the NN method with adaptive variance solution (NMSE $=0.10$ ), and for measurements.

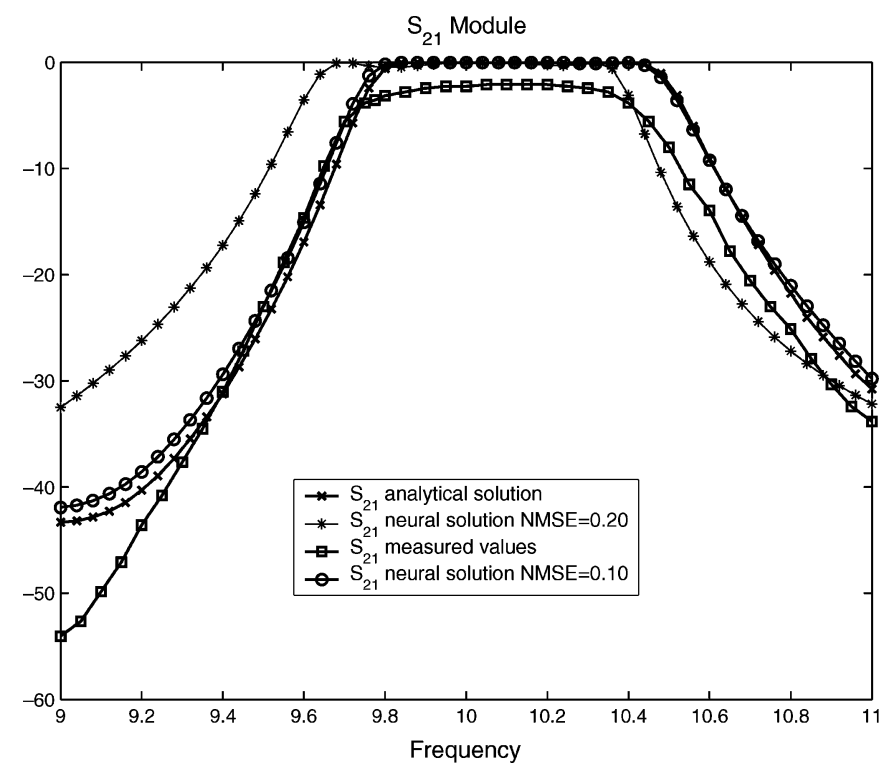

Fig. 11. $S_{21}$ module for the filter shown in Fig. 9. Results are shown for the direct solution, for the NN method with fixed variance solution (NMSE = $0.20)$, for the $\mathrm{NN}$ method with adaptive variance solution (NMSE $=0.10$ ), and for measurements.

neural strategies (see Fig. 9). While the first filter is composed of only coupled microstrip lines, the second filter connects the coupled lines sections with an extra length of transmission line, as shown in Fig. 9.

It should be pointed out that, for the analysis of this second structure, no training phase need to be applied. Consequently, the analysis of the second filter can proceed directly with the already trained RBFNNs, in a very fast way. This is because the box and the layered structure is the same as in the first example. In Figs. 10 and 11, the module of the $S_{11}$ - and $S_{21}$-parameters are shown. Measured results are also shown to validate the general neural CAD package. 


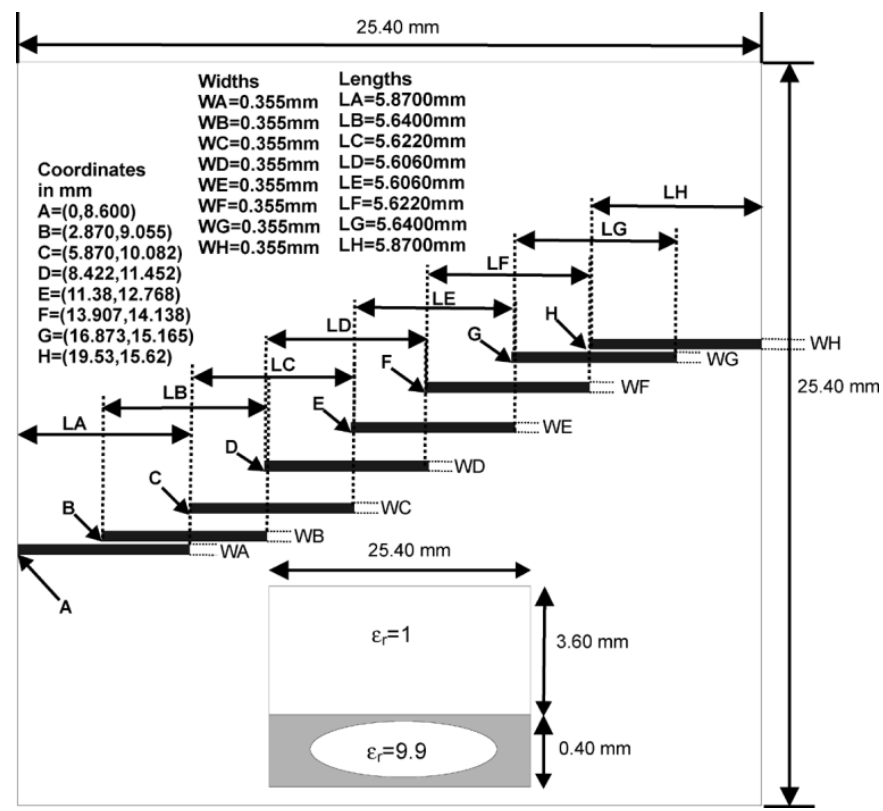

Fig. 12. Sixth-order bandpass filter designed with the NMSE $=0.1 \mathrm{NN}$.

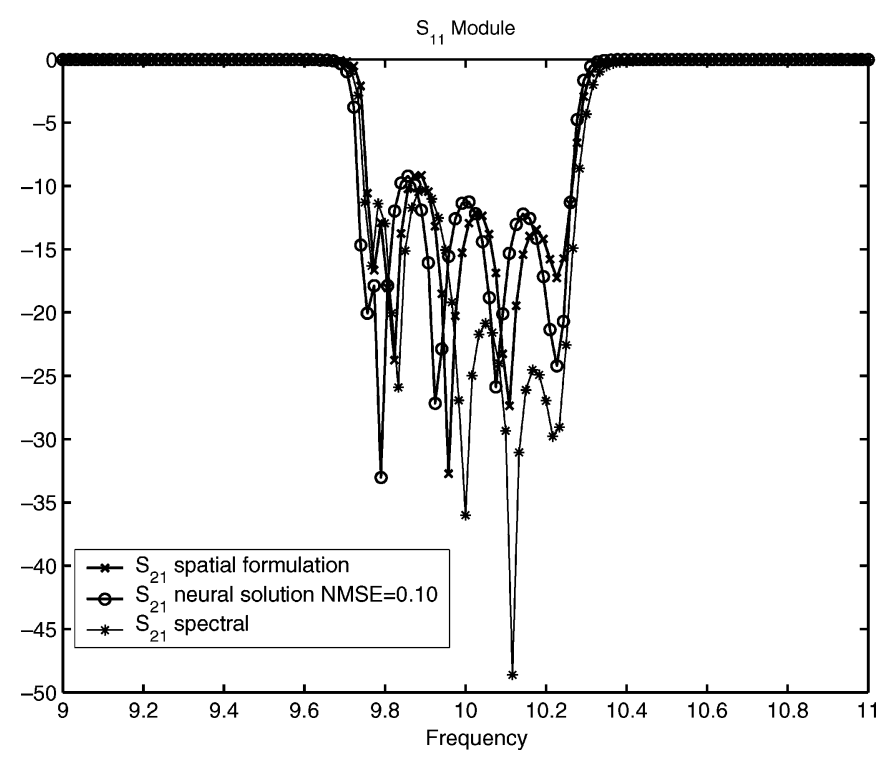

Fig. 13. $S_{11}$ module for the filter shown in Fig. 12. Results are shown for the direct solution, for the NN method with adaptive variance solution (NMSE $=$ 0.10 ), and with a different spectral domain technique.

It is interesting to notice that the RBFNNs with NMSE $=0.1$ have enough accuracy for the analysis and design of more complex bandpass filters. To show that this is indeed the case, we present an additional example with a sixth-order filter shown in Fig. 12, again in the same box as before. We present in Figs. 13 and 14 the scattering parameters of this more complex structure. In Figs. 13 and 14, we show the results obtained with the NN technique and with the direct evaluation of the Green's functions. We can observe very good agreement between both results. In order to validate the whole CAD package, we also include the results obtained with a different spectral domain technique [30]. In spite of using two very different numerical methods, we can observe good agreement in the recovered electrical performance of the filter.

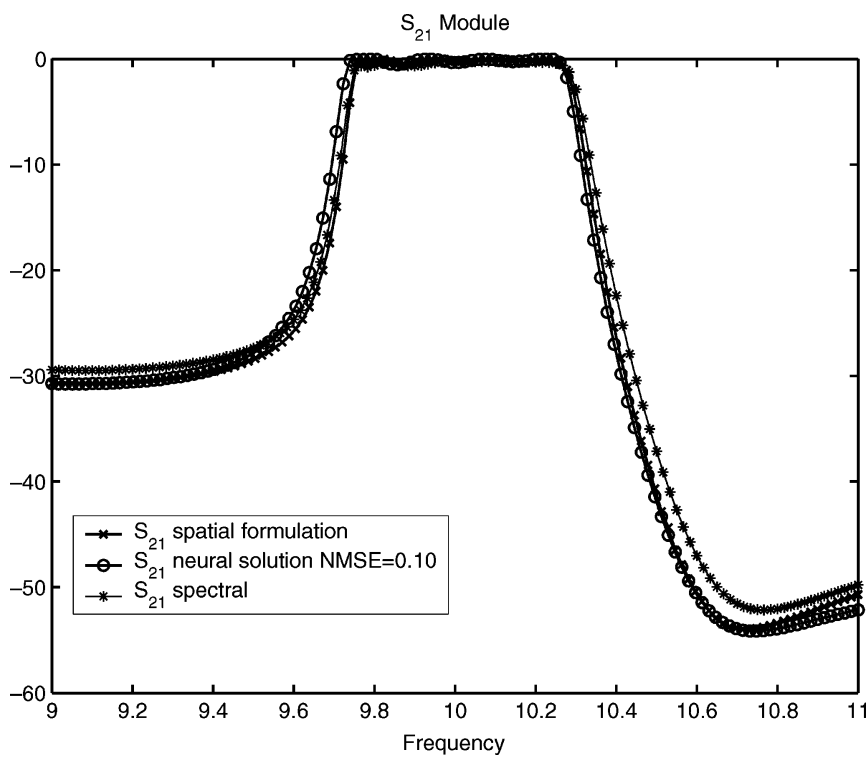

Fig. 14. $S_{21}$ module for the filter shown in Fig. 12. Results are shown for the direct solution, for the NN method with adaptive variance solution (NMSE = 0.10 ), and with a different spectral domain technique.

TABLE $\mathrm{V}$

ObSERVATION, Source, FREQuency REgION SELECTION Time, AND RBFNN Training Time When Fixed Variance STRATEgy is SELECTEd

\begin{tabular}{|c|c|c|c|c|c|}
\hline & $\begin{array}{c}\text { Observation } \\
\text { Region }\end{array}$ & $\begin{array}{c}\text { Source } \\
\text { Region }\end{array}$ & $\begin{array}{c}\text { Frequency } \\
\text { Region }\end{array}$ & $\begin{array}{c}\text { RBFNN } \\
\text { Training }\end{array}$ & $\begin{array}{c}\text { Total } \\
\text { Time }\end{array}$ \\
\hline \hline Time & $32^{\prime}$ & $3{\mathrm{~h} 20^{\prime}}^{\prime}$ & $35^{\prime}$ & $9 \mathrm{~h} 39^{\prime}$ & $14 \mathrm{~h} 6^{\prime}$ \\
\hline
\end{tabular}

We have shown with this example that the same RBFNN employed in the analysis of the first circuit can be used for the analysis of another printed structure, leading to similar degree of accuracy. The NN method has proven its ability to calculate the electrical circuit behavior of different printed circuits, provided the box and the layered structure remain unchanged.

As already discussed in this paper, the main advantage of the $\mathrm{NN}$ method is the fast calculation of the Green's functions once it is properly trained. The training of the RBFNN is performed for a given shielded structure with certain dimensions and dielectrics topology. The use of the RBFNN during the numerical solution of the IE allows for the analysis of complex circuits in a near real time fashion.

With respect to the computational time, two main stages can be distinguished in the NN design. First, the generation of the observation, source, and frequency regions, and, second, the training of the corresponding RBFNN. In Table V, the regions' selection time and the training RBFNN time are shown when the fixed variance strategy is used. A total of 19 RBFNNs, corresponding to the regions generated, were trained. The sum of these selection and training times amounts to the total initial fixed time needed, before the $\mathrm{NN}$ method can be applied inside the IE for the analysis of practical circuits. In Table VI, the regions' selection time and the training RBFNN time are shown when the adaptive variance strategy is used. A total number of 73 RBFNNs were trained this time. Once the RBFNN training phase has finished, the IE technique is applied in a fast way. In Table VII, we present the computational time needed for the solution of the IE for the two first filters presented. As can be seen from Table VII, the NN method is several hundred times faster 
TABLE VI

ObSERVATION, SOURCE, FrEQUENCY REgION SELECTION TIME, AND RBFNN Training Time When AdAPtive VARIANCE STRATEGY IS SElEcted

\begin{tabular}{|c||c|c|c|c|c|}
\hline & $\begin{array}{c}\text { Observation } \\
\text { Region }\end{array}$ & $\begin{array}{c}\text { Source } \\
\text { Region }\end{array}$ & $\begin{array}{c}\text { Frequency } \\
\text { Region }\end{array}$ & $\begin{array}{c}\text { RBFNN } \\
\text { Training }\end{array}$ & Total Time \\
\hline \hline Time & 2h 23' & $10 \mathrm{~h} \mathrm{33^{ \prime }}$ & $41^{\prime}$ & $63 \mathrm{~h} 27^{\prime}$ & $77 \mathrm{~h} \mathrm{4'}$ \\
\hline
\end{tabular}

TABLE VII

COMPARISON BETWEEN THE DIRECT IE SOLUTION AND THE NN IE SOLUTION TO COMPUTE THE $S$-PARAMETERS IN 51 FREQUENCY POINTS

\begin{tabular}{|c|c|c|c|c|c|}
\hline Filter & $\begin{array}{c}\text { Direct } \\
\text { Solution }\end{array}$ & $\begin{array}{c}\text { Fixed } \\
\text { Variance } \\
\text { Strategy }\end{array}$ & $\begin{array}{c}\text { Times } \\
\text { Faster }\end{array}$ & $\begin{array}{c}\text { Adaptive } \\
\text { Variance } \\
\text { Strategy }\end{array}$ & $\begin{array}{c}\text { Times } \\
\text { Faster }\end{array}$ \\
\hline \hline Filter 1 & $43 \mathrm{~h} \mathrm{58^{ \prime }}$ & $515 \mathrm{~s}$ & 307 & $715 \mathrm{~s}$ & 216 \\
\hline Filter 2 & $39 \mathrm{~h} 32^{\prime}$ & $451 \mathrm{~s}$ & 315 & $635 \mathrm{~s}$ & 222 \\
\hline
\end{tabular}

than the exact solution method. The initial time when the fixed variance strategy is used is $14 \mathrm{~h}$ and $6 \mathrm{~min}$. In the case of the adaptive variance method, this initial time amounts to $77 \mathrm{~h}$ and $4 \mathrm{~min}$. We have to remark that these are initial time values. After the training, any printed circuit (as the three practical examples shown in this paper) is analyzed in a few seconds per frequency point. Moreover, any optimization technique used to improve the circuit performance can be applied, obtaining the results of each analysis in a few seconds per frequency point. This shows clearly the usefulness of the neural technique proposed in this paper.

Table VII shows the time needed to compute the scattering parameters for 51 frequency points. The IE technique using the RBFNN, trained with the fixed variance strategy, takes $10.09 \mathrm{~s}$ per frequency point to calculate the scattering parameters of the first filter and $8.84 \mathrm{~s}$ for the second filter. On the other hand, the IE technique using the RBFNN, trained with the adaptive variance strategy, spends $14.02 \mathrm{~s}$ per frequency point to compute the scattering parameters of the first filter and $12.45 \mathrm{~s}$ for the second filter. In both cases, the analysis calculated when the variance is fixed is slightly faster than when the adaptive variance is used. To explain this, we have to keep in mind that every time a source-observation pair is presented to an RBFNN, the output calculation time depends only on the size of the particular RBFNN. Since the adaptive variance method is adjusted to obtain lower errors, then every RBFNN contains a slightly larger number of neurons. The small difference in time between both solutions is not important in comparison with the error level achieved when the adaptive variance is used, which cannot be obtained with the fixed variance strategy. All of the results presented in the paper were obtained on a Pentium IV computer with a 3.06-GHz processor and a total RAM memory of 2 GB.

\section{CONCLUSION}

Two novel strategies, based on NNs, are used to analyze printed circuits in shielded microwave structures. The NN employed is the RBFNN. The RBFNN is trained to approximate the spatial domain Green's functions of the relevant potentials needed in the IE technique. The first strategy divides the whole input space into an arbitrary number of source, observation, and frequency regions. This separation allows a better training and an improvement in the fulfillment of the boundary conditions for the potentials at the cavity walls. The second strategy combines the previous region subdivision with an adaptive algorithm for the selection of the optimum spatial variance. We have shown that this method leads to lower error levels, and consequently to a better microwave circuit analysis. Once all the RBFNNs have been trained, the NNs can replace the direct Green's functions solution allowing fast circuit analysis. The IE technique combined with the NN method is several hundred times faster than the direct solution. This remarkable time gain makes the designing and training times negligible. Consequently, the NN method presented is a useful method that can be integrated into a CAD tool, for the analysis, design, and optimization of practical shielded MMIC devices.

\section{REFERENCES}

[1] H.-W. Chiu, S.-S. Lu, and Y.-S. Lin, “A 2.17-dB NF 5-GHz-band monolithic cmos lna with 10-mw DC power consumption," IEEE Trans. Microw. Theory Tech., vol. 53, no. 3, pp. 813-824, Mar. 2005.

[2] G. Virone, R. Tascone, M. Baralis, O. A. Peverini, A. Olivieri, and R. Orta, "A novel design tool for waveguide polarizers," IEEE Trans. Microw. Theory Tech., vol. 53, no. 3, pp. 888-894, Mar. 2005.

[3] T.-H. Loh and C. Mias, "Implementation of an exact modal abosorbing boundary condition for the application of the finite element time domain technique to discontinuity problems in closed homogeneous waveguide," IEEE Trans. Microw. Theory Tech., vol. 52, no. 3, pp. 882-888, Mar. 2004

[4] S.-G. Pan and I. Wolff, "Scalarization of dyadic spectral Green's functions and network formalism for three-dimensional full-wave analysis of planar lines and antennas," IEEE Trans. Microw. Theory Tech., vol. 42, no. 11, pp. 2118-2127, Nov. 1994.

[5] A. A. Melcon, J. R. Mosig, and M. Guglielmi, "Efficient CAD of boxed microwave circuits based on arbitrary rectangular elements," IEEE Trans. Microw. Theory Tech., vol. 47, no. 7, pp. 1045-1058, Jul. 1999.

[6] A. A. Melcon and J. R. Mosig, "Two techniques for the efficient numerical calculation of the Green's functions for planar shielded circuits and antennas," IEEE Trans. Microw. Theory Tech., vol. 48, no. 9, pp. 1492-1504, Sep. 2000.

[7] S. M. Rao, D. R. Wilton, and A. W. Glisson, "Electromagnetic scattering by surfaces of arbitrarily shape," IEEE Trans. Antennas Propag., vol. AP-30, no. 5, pp. 409-418, May 1982.

[8] C. Christodoulou and M. Georgipoulos, Applications of Neural Net works in Electromagnetics. Norwell, MA: Artech House, 2001.

[9] F. Wang and Q. J. Zhang, "Knowledge-based neural models for microwave design," IEEE Trans. Microw. Theory Tech., vol. 45, no. 12, pp. 2333-2343, Dec. 1997.

[10] M. Vai and S. Prasad, "Microwave circuit analysis and design by a massively distributed computing network," IEEE Trans. Microw. Theory Tech., vol. 43, no. 5, pp. 1087-1094, May 1995.

[11] T. Horng, C. Wang, and N. G. Alexopoulos, "Microstrip circuit design using neural networks," in IEEE MTT-S Int. Microwave Symp. Dig., 1993, pp. 413-416.

[12] P. M. Watson and K. C. Gupta, "Design and optimization of cpw circuits using EM-ANN models for cpw components," IEEE Trans. Microw. Theory Tech., vol. 45, no. 12, pp. 2515-2523, Dec. 1997.

[13] A. H. Zaabab, Q. J. Zhang, and M. S. Nakhle, "Neural network modeling approach to circuit optimization and statistical design," IEEE Trans. Microw. Theory Tech., vol. 43, no. 6, pp. 1349-1358, Jun. 1995.

[14] F. Wang, V. K. Devabhaktuni, and Q. J. Zhang, "A hierarchical neural network approach to the development of a library of neural models for microwave design," IEEE Trans. Microw. Theory Tech., vol. 46, no. 12, pp. 2391-2403, Dec. 1998.

[15] G. L. Creech, B. J. Paul, C. Lesniak, T. J. Jenkins, and M. C. Calcatera "Artificial neural networks for fast and accurate EM-CAD of microwave circuits," IEEE Trans. Microw. Theory Tech., vol. 45, no. 5, pp. 794-802, May 1997

[16] E. A. Soliman, M. A. El-Gamal, and A. K. Abdelmageed, "Neural network model for the efficient calculation of Green's fucntions in layered media," Int. J. RF Microwave Computer-Aided Eng., vol. 13, pp. 128-135, Mar. 2003.

[17] J. J. Yang, Y. L. Chow, G. E. Howard, and D. G. Fang, "Complex image of an electric dipole in homogeneous and layered dielectrics between two ground planes," IEEE Trans. Microw. Theory Tech., vol. 40, no. 3, pp. 595-599, Mar. 1992.

[18] R. K. Mishra and A. Patnaik, "Neurospectral computation for complex resonant frequency of microstrip resonators," IEEE Microw. Guided Wave Lett., vol. 9, no. 9, pp. 351-453, Sep. 1999. 
[19] - "Neurospectral computation for input impedance of rectangular microstrip antenna," Electron. Lett., vol. 35, pp. 1691-1693, Sep. 1999

[20] — - "Designing rectangular patch antenna using the neurospectral method," IEEE Trans. Antennas Propag., vol. 51, no. 8, pp. 1914-1921, Aug. 2003.

[21] J. P. Garcia, D. C. Rebenaque, F. D. Q. Pereira, J. L. G. Tornero, and A. A. Melcon, "Fast and efficient calculation of the multilayered shielded Green's functions employing neural networks," Microwave Opt. Technol. Lett., vol. 44, pp. 61-66, Nov. 2004.

[22] J. P. Garcia, F. D. Q. Pereira, D. C. Rebenaque, J. L. G. Tornero, and A. A. Melcon, "A neural network method for the multilayered shielded Green's function calculation," in Proc. 34th Eur. Microwave Conf., Oct. 2004, pp. 841-844.

[23] _ "A neural network method for the analysis of multilayered shielded microwave circuits," in IEEE MTT-S Int. Microwave Symp. Dig., Jun. 2005, pp. 1601-1604.

[24] V. Devabhaktuni, M. Yagoub, and Q. Zhang, "A robust algorithm for automatic development of neural-network models for microwave applications," IEEE Trans. Microw. Theory Tech., vol. 49, no. 12, pp. 2282-2291, Dec. 2001.

[25] S. Haykin, Neural Networks, A Comprehensive Foundation. Upper Saddle River, NJ: Prentice-Hall, 1999.

[26] A. A. Melcon and J. R. Mosig, "The summation-by-parts algorithm-A new efficient technique for the rapid calculation of certain series arising in shielded planar structures," IEEE Trans. Microw. Theory Tech., vol. 50, no. 1, pp. 215-218, Jan. 2002.

[27] S. Chen, C. F. N. Cowan, and P. M. Grant, "Orthogonal least squares learning algorithm for radial basis function networks," IEEE Trans. Neural Netw., vol. 2, no. 2, pp. 302-309, Mar. 1991.

[28] S. Chen, P. M. Grant, and C. F. N. Cowan, "Orthogonal least squares algorithm for training multi-output radial basis function networks," Proc. Inst. Elect. Eng.-Radar and Signal Process., pt. F, vol. 139, no. 6, pp. 378-384, Dec. 1992.

[29] A. Sherstinsky and R. W. Picard, "On the efficiency of the orthogonal least squares training method for radial basis fucntion networks," IEEE Trans. Neural Netw., vol. 7, no. 1, pp. 195-200, Jan. 1996.

[30] R. Faraji-Dana and Y. L. Chow, "Accurate and efficient CAD tool for the design of optimum packaging for (M)MIC's," Proc. Inst. Elect. Eng.-Microwave Antennas Propag., vol. 142, pp. 81-88, Apr. 1995.

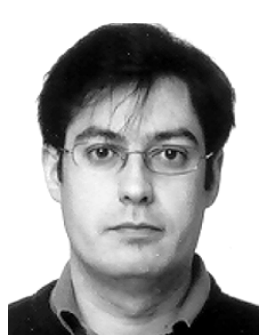

Juan Pascual García (S’05) was born in Castellón, Spain, in 1975. He received the Telecommunications Engineer degree from the Technical University of Valencia, Valencia, Spain, in 2001, and is currently working toward the Ph.D. degree at the Technical University of Cartagena (UPCT), Cartagena, Spain.

In 2002, he joined the CEMI Research Center, UPCT, as a Research Assistant. In 2003, he joined the Communications and Information Technologies Department, UPCT. His research interests include NNs, genetic algorithms, and their applications in the analysis and development of a CAD tool for microwave circuits and antennas.

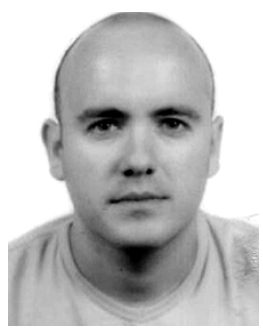

Fernando Quesada Pereira (S'05) was born in Murcia, Spain, in 1974. He received the Telecommunications Engineer degree from the Technical University of Valencia (UPV), Valencia, Spain, in 2000, and is currently working toward the Ph.D. degree at the Technical University of Cartagena (UPCT), Cartagena, Spain

In 1999, he joined the Radiocommunications Department, UPV, as a Research Assistant, where he was involved in the development of numerical methods for the analysis of anechoic chambers and tag antennas. In 2001, he joined the Communications and Information Technologies Department, UPCT, initially as a Research Assistant, and then as an Assistant Professor. In 2005, he spent six months as a Visiting Scientist with the University of Pavia, Pavia, Italy. His current scientific interests include IE numerical methods for the analysis of antennas and microwave devices.

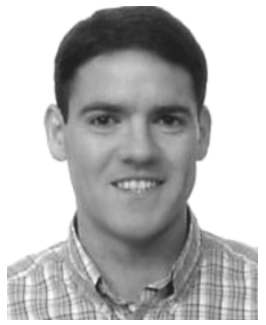

David Cañete Rebenaque (S'04) was born in Valencia, Spain, in 1976. He received the Telecommunications Engineer degree from the Technical University of Valencia, Valencia, Spain, in 2000, and is currently working toward the Ph.D. degree at the Technical University of Cartagena (UPCT), Cartagena, Spain.

During 2001, he was an RF Engineer with a mobile communications company. In 2002, he joined the Communications and Information Technologies Department, UPCT. His research interests include the analysis and design of microwave circuits and active antennas.

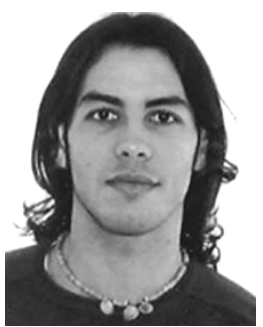

José Luis Gómez Tornero was born in Murcia, Spain, in 1977. He received the Telecommunications Engineer degree from the Polytechnic University of Valencia (UPV), Valencia, Spain, in 2001, and is currently working toward the Ph.D. degree at the Technical University of Cartagena (UPCT), Cartagena, Spain.

In 1999, he joined the Radiocommunications Department, UPV, as a research student, where he was involved in the development of analytical and numerical tools for the study and automated design of microwave filters in waveguide technology for space applications. In 2000, he joined the Radio Frequency Division, Industry Alcatel Espacio, Madrid, Spain, where he was involved with the development of microwave active circuits for telemetry, tracking, and control (TTC) transponders implicated in many different spatial missions for the European Space Agency (ESA), the National Aeronautics Space Administration (NASA), and other space agencies. In 2001, he joined the Communications and Information Technologies Department, UPCT, as an Assistant Professor, where he is currently involved with teaching activities. His scientific research is focused on the analysis and design of leaky-wave antennas for millimeter-wave-band applications and the development of numerical methods for the analysis of novel passive radiating structures in planar and waveguide technologies. His scientific interests also include the study of active devices for microwave and millimeter-wave bands, such as oscillators and active antennas.

Prof. Gómez Tornero was the recipient of the Second National Award presented by the EPSON-Ibérica Foundation in 2004 for the best doctoral project in the field of technology of information and communications.

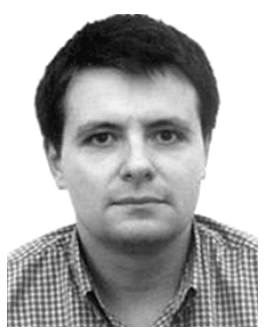

Alejandro Alvarez Melcón (M’99) was born in Madrid, Spain, in 1965. He received the Telecommunications Engineer degree from the Polytechnic University of Madrid (UPM), Madrid, Spain, in 1991, and the Ph.D. degree in electrical engineering from the Swiss Federal Institute of Technology, Lausanne, Switzerland, in 1998.

In 1988, he joined the Signal, Systems, and Radiocommunications Department, UPM, as a research student, where he was involved with the design, testing, and measurement of broad-band spiral antennas for electromagnetic measurements support (EMS) equipment. From 1991 to 1993, he waws with the Radio Frequency Systems Division, European Space Agency (ESA/ESTEC), Noordwijk, The Netherlands, where he was involved in the development of analytical and numerical tools for the study of waveguide discontinuities, planar transmission lines, and microwave filters. From 1993 to 1995, he was with the Space Division, Industry Alcatel Espacio, Madrid, Spain, and was with the ESA, where he collaborated in several ESA/ESTEC contracts. From 1995 to 1999, he was with the Swiss Federal Institute of Technology, École Polytechnique Fédérale de Lausanne, Lausanne, Switzerland, where he was involved in the field of microstrip antennas and printed circuits for space applications. In 2000, he joined the Communications and Information Technologies Department, Technical University of Cartagena, Cartagena, Spain, where he is currently involved with teaching and research activities.

Dr. Alvarez Melcón was the recipient of the Journée Internationales de Nice sur les Antennes (JINA) Best Paper Award for the best contribution to the JINA'98 International Symposium on Antennas and the Colegio Oficial de Ingenieros de Telecomunicación (COIT/AEIT) Award for the best Ph.D. thesis in basic information and communication technologies. 